\title{
A SURVEY OF REAL-TIME ROUTING PROTOCOLS FOR WIRELESS SENSOR NETWORKS
}

\author{
Sandhya Rachamalla ${ }^{1}$ Dr. Anitha Sheela Kancharla ${ }^{2}$ \\ ${ }^{1}$ Department of Electronics and Communications Engineering, Sreenidhi Institute of \\ Science and Technology, Hyderabad, Andhra Pradesh, India \\ ${ }^{2}$ Department of Electronics and Communications Engineering, JNTUH, Hyderabad, \\ Andhra Pradesh, India \\ reddysandhya28@gmail.com
}

\begin{abstract}
Sensor networks is an interdisciplinary research area that draws on contributions from signal processing, wireless networking and associated routing protocols, database management and information systems, distributed algorithms and MEMS technology. Recent developments in Wireless Sensor Networks have resulted in wide variety of real-time applications. Many real-time routing protocols are designed to meet the requirements of these applications where timely delivery of the sensed information is given the top priority. This paper presents the comparative analysis of various existing real-time routing protocols for wireless sensor networks, which emphasizes on various factors like end-to-end delay, energy, mobility, scalability and highlighted various challenges for future research.
\end{abstract}

\section{KEYWORDS}

Wireless Sensor Networks (WSNs), Micro-Electro Mechanical Systems (MEMS), Real-time routing (RT), Routing protocols (RPS), QoS, Dead line miss ratio (DMR), Energy consumption, Void avoidance, end-toend delay

\section{INTRODUCTION}

Advances in wireless networking and MEMS technology have enabled a new generation of massive-scale sensor networks suitable for wide range of applications. Unlike a centralised system, a sensor network is subject to a unique set of resource constraints such as battery power, limited network bandwidth and a small amount of memory for signal processing and task scheduling. Applications of sensor networks are wide range and can vary significantly in application requirements, sensing modality and node deployment. Some of the applications are environmental data collection, habitat exploration of animals, Security monitoring, guiding systems of missiles, detection of hidden weapons, patient diagnosis, flood detection, infrastructure protection, battle field awareness, home automation, context aware computing.

Examples of real time sensor applications can be found in many commercial systems, military, surveillance systems and safety critical systems [1]. In target tracking applications of Wireless Sensor Networks the collected data must reach the base station or control unit in predefined time to ensure an effective real time tracking of the required sensed region [2].In medical applications, sensed data can be sent regularly over the network to the automated monitoring systems which are designed to alert the concerned doctor [3]. In these applications, timely and reliable data delivery is very important for positive results. Designing of the real-time routing protocol for 
WSN is a challenging task as WSN is energy constrained due to the tiny battery power while RTs demand more power. Both of these constraints, energy and time, are inversely proportional to each other. On-time delivery of sensed information requires maximum transmission power.

The MAC layer mechanisms deliver the information by considering real time needs, but effect remains locally. Therefore the real-time routing protocol has to guarantee on-time delivery globally. Hence, the designing of a real-time routing protocol for WSNs with optimum energy consumption is a challenging task. In paper [4], authors stated various design issues like data delivery model, node deployment, energy consumption, fault tolerant, scalability and other QoS to be considered while designing the real time routing protocol.

\section{Real- Time Routing Protocols}

Many researchers have provided solutions for real-time routing in WSNs. This section provides the comparative analysis of the various existing real time routing protocols for WSNs emphasizing their strengths and weaknesses and various other challenges. Real time routing is discovering an optimum route from source to destination which meets the real time constraints. Timely and reliable data delivery is very important for positive results as out-dated data may lead to disaster effects.

\subsection{Ad hoc On-Demand Distance-Vector (AODV) Routing algorithm}

AODV [5] is an on-demand routing protocol which builds route between the nodes only when the source node demands for routing the sensed data. And as long as required by the source node the routes are maintained. This routing algorithm provides the tree formation connecting the multicast members. It uses the sequence numbers to ensure the freshness of the routes resulting in loop-free routing.

In this protocol, as shown in Figure 1 (a), RouteRequest packets are broadcasted by the source node when the route is not available from source to the destination. From this it may obtain multiple routes to the destination for a single RouteRequest. A sequence number called destination sequence number (DestSeqNum) is used in RouteRequest packets to determine the latest path to the destination. After receiving RouteRequest packet with DestSeqNum, an intermediate node verifies it and updates only if received sequence number is greater than the last stored one. And an intermediate node upon receiving a RouteRequest, it either forwards it or prepares a RouteReply, as shown in Figure 1 (b), if it already has a valid route to the destination. The validity of a route is determined at the intermediate node by comparing the sequenced numbers with the received destination sequence number. Duplicate copies of RouteRequest packets having same sequence number are discarded at intermediate node. All intermediate nodes having the valid routes to the destination are allowed to send the RouteReply packets to the source.

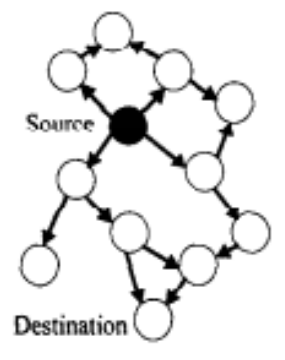

(a)

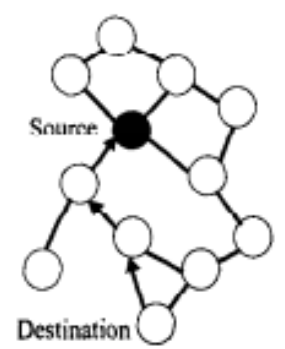

(b)

Figure 1: The (a) RouteReqest and (b) RouteReply message cycles [6]. 
The main advantage of this protocol is that it is a reactive protocol and routes are established ondemand i.e. whenever source wants to deliver the sensed data to destination then only the path is established. Sequence numbers are used to find the latest routes to the destination. The disadvantage is that the intermediate nodes can lead to inconsistent routes if the sequence number is old. Also periodic beaconing leads to unnecessary bandwidth consumption. Also it does not repair a broken path locally. The connection setup delay is less, but control overhead is heavy. This is a reactive protocol which maintains the routing information for a small subset of destinations, namely for those in use. If there is no route for a new destination, a route discovery process is invoked, which leads to the significant delays in sensor networks. This limitation makes this on- demand algorithm less suitable for real-time applications.

\subsection{Dynamic Source Routing (DSR) Protocol}

DSR [7] protocol is a reactive protocol and another on-demand routing protocol. Unlike AODV, it is designed to restrict the bandwidth consumed by the control packets in WSNs. It eliminates the periodic updating of routing tables as it is beacon-less.

Source node broadcasts a RouteRequest packet if it does not have a route for destination and the packet is further forwarded by each intermediate node if it has not forwarded previously or if it is not destination node. A Sequence number is carried by each RouteRequest packet generated by the source node throughout the path it has traversed. Each intermediate node after receiving the RouteRequest packet checks the sequence number and is forwarded only if it is not a duplicate RouteRequest with same sequence number. In this way the sequence numbers help in avoiding loops in routing paths and multiple transmissions of the RouteRequest by an intermediate node. The destination node receives the RouteRequest packet and replies to the source node through the reverse path the RouteRequest packet had traversed. Route cache is used which stores all possible routing information taken from data packet originated from the source node. After receiving a RouteRequest, if an intermediate node has a route to the destination node in its route cache it replies to the source node the entire route information from the source node to the destination node. The source node may receive multiple replies from intermediate nodes, but it selects the latest and best route. Each data packet carries the complete route information to its destination.

The main advantage of this reactive routing protocol is that there is no need for the update of the messages. The route cache information in the intermediate nodes efficiently reduces the overhead. The disadvantage is that it cannot repair broken link locally as the complete path is originated from the source node. In case of broken link, the source node finds the new route only after receiving the RouteError from the node adjacent to the broken link. Also route cache information may result in inconsistent route setup. The connection setup delay is high. Due to the source routing mechanism, considerable routing overhead is involved which is directly proportional to the path length. Delay in discovering new routes and considerable connection set up delay makes this protocol less suitable for real time applications.

\subsection{RAP (A Real time communication architecture for large scale wireless sensor networks)}

RAP [8] is the first real-time communication architecture that handles the deadline issues pertaining to large scale WSNs. It uses the high level query and event services and the velocity monotonic scheduling (VMS) policy to schedule packets.

RAP provides APIs (Application programming interface) for the applications to address their queries. This layered architecture ensures that the query is sent to all the nodes in the specified area, and the responses to the queries are sent back to the base station. The protocol stack of RAP consists of location addressed protocol (LAP) in the transport layer, Velocity monotonic 
scheduling (VMS) as the geographic routing protocol, and a contention based MAC scheme to support prioritization as shown in the Figure 2.

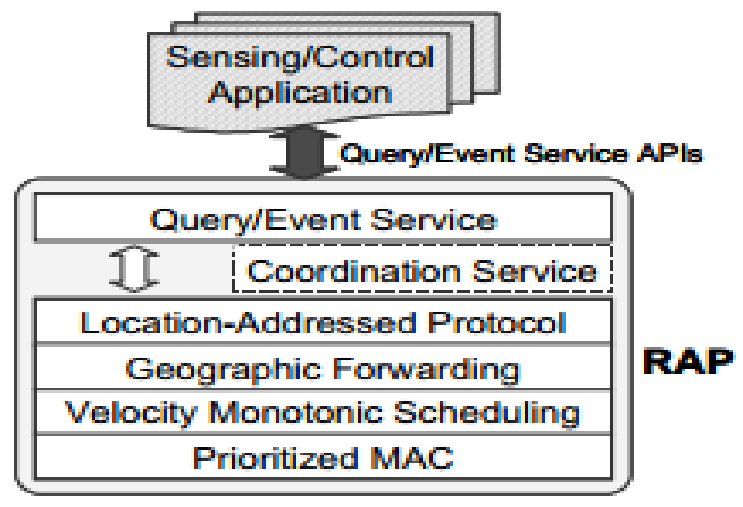

Figure 2: RAP communication architecture model [8]

LAP is a connectionless transport layer protocol. The location based addressing is used for nodes instead of a unique addressing scheme such as IP address or unique ID for all nodes. The unique addressing mode is difficult to address the nodes in a sensor network having large number of nodes. And it supports three kinds of communications mechanisms: unicast, area multicast, and area anycast. VMS calculates the requested velocity, which takes into consideration the timing and the distance constraints i.e. it is with this velocity the packet is required to travel towards the destination. The requested velocity is a measure of the urgency of the packet. If a packet can travel at its requested velocity, that is, can cover the required distance within a specified time, then it can meet its deadline. VMS gives high priority to the packets which have higher requested velocities. Dynamic VMS recalculates the velocity at each intermediate node. This can be mapped onto a MAC layer priority, handled by the contention-based MAC layer.

Geographic forwarding is used and hence the scalability is possible. One of the performance metrics of this architecture is mobility. The notation of the velocity is exploited in real-time communication protocols on sensor networks by this architecture. The key constraints in sensor networks, namely end-to-end dead line and communication distance are the factors considered in this protocol. This kind of routing protocol cannot handle long term congestion where diversion of routing is necessary away from hotspot. The protocol hence provides convenient services for the application layer programs that require real-time support.

\subsection{SPEED (A Stateless Protocol for Real-Time Communication in Sensor Networks)}

SPEED [9] protocol is an important real-time communication protocol to route packets with the desired speed for sensor networks. This protocol provides the real-time communication services, such as real-time unicast, real-time area-multicast, real-time area-anycast. These three types of communication patterns are associated with the type of data delivery. If a part of the network is to be sensed and needs to be reported to the base station, then it is called as unicast. If it motivates another type of communication pattern where one end of the route may be an area rather than an individual node, then it is known as area-multicast. Sometimes it is sufficient to have any node to respond in an area to avoid redundant information being carried by the nodes in the network. We can call this routing service as area-anycast. The SPEED is a localized and stateless protocol which carries minimal control overhead. This protocol is provisioning the efficiency in real-time communication with the desired speed being maintained across the network for the data packets 
from source to the destination through a novel combination of feedback control and nondeterministic QoS aware geographic forwarding.

The SPEED Protocol architecture is shown in the Figure 3. This architecture provides the combination of MAC and network layer procedures which improves the end-to-end delay maintaining desired speed throughout the network and provides good response to the congestion by reducing the traffic near 'hotspots' with back pressure rerouting. It consists of the following components:

- An API( Application Programming Interface)

- A neighbour beacon exchange scheme

- A delay estimator

- The stateless Non-deterministic Geographic forwarding algorithm (SNGF)

- A neighbourhood Feedback loop (NFL)

- Backpressure Rerouting

- Last mile processing

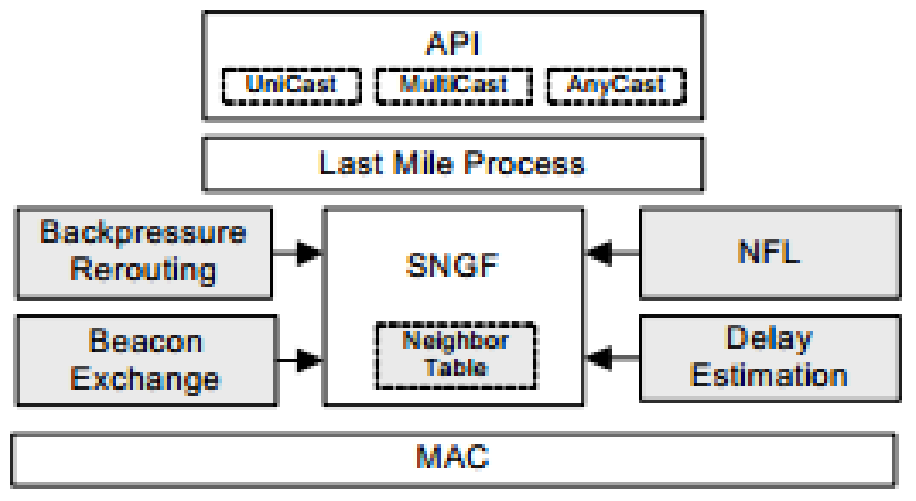

Figure 3: SPEED Protocol [9]

As shown in Figure 3, SNGF is an algorithm used in this protocol to select the suitable neighbour node that can support the required speed. NFL and Back pressure Rerouting are the two modules responsible for diverting the traffic when congestion occurs. The last mile process is there to support the three communication patterns mentioned before. Delay estimator calculates the round trip single hop delay and newly measured delay is compared with previous delays. In paper [9], the authors argue that this delay estimation is better metric to represent the congestion than the average queue size. Beacon exchange provides the geographic location of the neighbour nodes so that SNGF can do geographic based routing.

This protocol also handles void problems. However, the protocol maintains single speed for packet delivery throughout the network, which is not suitable for sending various types of data packets having different deadline. It doesn't consider the energy metric.

\subsection{MMSPEED (Multi-path multi-speed protocol for QoS guarantee of Reliability \& Timeliness in WSN)}

MMSPEED [10] extends the SPEED protocol to support different velocities and level of reliability for multiple probabilistic QoS guarantee in WSNs. The QoS provisioning is performed in two quality domains, namely timeliness and reliability. Unlike the SPEED, as shown in Figure 4 , this protocol provides the multiple network wide options to obtain the QoS in terms of 
timeliness. For timeliness, this protocol provides multiple layers of network wide speeds augmented by the two novel techniques: Virtual isolation and dynamic compensation.

Virtual isolation provides the classification of incoming packets according to their speed classes and places them in appropriate queues, which supports for fast delivery of high-speed packets, not being affected by the low speed packets. The packets are served in FIFS manner according to the priority in the queue. For achieving the QoS in terms of reliability, MM-SPEED provides the probabilistic multipath forwarding. For the prioritized transmission between the nodes, the network layer has to take support from MAC layer. Hence it uses dynamic compensation which adjusts the local decisions to meet their end-to-end deadlines. In order to provide the QoS differentiation in the reliability domain, it provides the multiple redundant paths to the final destinations, that is, the more the paths to the destination, the higher the probability that the packet reaches the destination.

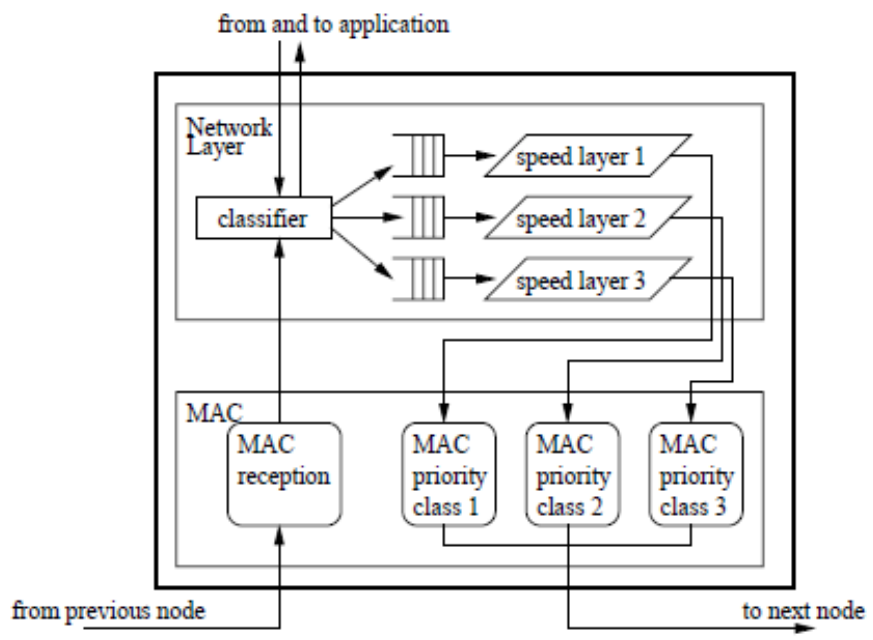

Figure 4: Protocol structure at sensor node [10]

This protocol provides the desirable properties such as scalability for large scale networks, self adaptability to the network dynamics, and works well for both urgent aperiodic and periodic packets. Many features of MMSPEED may lead to more energy consumption and frames with large overhead.

\subsection{RPAR (Real time Power Aware Routing protocol)}

RPAR [11] is the advance version of RAP. It is the only protocol that is designed to support the real time routing for WSNs with power control. Application specific communication delays are handled in this protocol by dynamically adapting transmission power and routing decisions based on the workload and packet deadlines. RPAR uses forwarding policy with power awareness and neighbourhood manager that efficiently discovers eligible neighbourhood node to forward the packet in wireless sensor networks. The key feature of this protocol is its adaptability, i.e. for tight deadlines, it trades energy and capacity to meet the desired time constraints, and for loose deadlines, it lowers the transmission power to increase the throughput. The protocol architecture consists of four modules as follows

- Dynamic velocity assignment module, which maps a packet deadline to a required packet velocity. When a node wants to forward a packet, it uses the velocity assignment module to calculate the required velocity based on the remaining distance between the present 
node and the destination node and time to live (TTL). It also prioritizes the packet based on their deadlines.

- Delay estimator module, which calculates the one- hop delay of different forwarding choices.

- Forwarding module makes forwarding decisions on packet-by-packet basis. It forwards the packet to the most energy efficient forwarding choice that meets the required velocity of the packet.

- Neighbourhood manager effectively handles the neighbourhood table and provides the best neighbor for forwarding the packets. If there is no information in neighborhood table about eligible forwarding node then the neighborhood manager is invoked to discover the forwarding choices with two mechanisms- power adaptation and neighbor discovery. In the power adaptation scheme, the transmission power is increased to meet the required velocity for the existing neighbors in the neighborhood table. In neighbor discovery scheme, new neighbors are discovered that meet the required velocity by sending the Route to Request (RTR) packets to the neighbors

The proposed power adaption and neighborhood mechanisms are on-demand and hence this protocol is a reactive protocol. This reactive approach help in discovering neighbors quickly with low control overhead.This protocol addresses important practical issues like broken links, scalability and bandwidth constraints. Performance results show that this protocol performs well in terms of energy consumption and deadline miss ratio.

\subsection{THVR (Two-Hop Velocity based routing protocol)}

THVR [12] is a two-hop neighbourhood information-based routing protocol for real time wireless sensor networks proposed to support the QoS in terms of real-time packet delivery along with better energy efficiency. In this protocol, two-hop information is used to determine the required velocity and routing decisions are made based on the two-hop velocity with probabilistic packet dropping mechanism for energy balancing. With the two-hop mechanism, the number of hops required to transmit the data from source to the destination decreases significantly.

THVR is comprised of four components

- Two-hop velocity based forwarding strategy

- Delay estimation scheme

- Energy- efficient probabilistic drop

- Optimal residual energy cost function for energy balancing.

By the two-hop information, congestion in the network could be predicted at an early time. The idea of two-hop information provides a trade off between performance and complexity. The packet deadline is mapped with an adopted velocity as in SPEED. This feature is well suited for delay constraints. The routing decision is based on the novel two-hop velocity along with the energy balancing mechanism. The key features of this protocol is that it achieves low deadline miss ratio along with the high energy efficiency by using novel two hop information based routing. This real time protocol also considers the efficient energy utilization that has not been addressed in SPEED and MM-SPEED. By using the two-hop packet delay estimation getting compared with the required velocity the forwarder node is selected. If suitable forwarder is not there then the packet is dropped with the probabilistic mechanism. THVR requires more neighbourhood information for a better decision increasing the complexity cost. 


\subsection{PATH (A Novel Real-Time Power Aware Two-Hop Routing Protocol for Wireless Sensor Networks)}

PATH [13] is a newly proposed real-time protocol which uses the two-hop neighbour information for routing decisions. The real-time performance is improved by means of reducing the packet dropping in routing decisions. Dynamic adjustment of transmission power is adopted to reduce the probability of packet dropping thereby increasing the number of transmission packets that can meet their deadline. In THVR and SPEED, the main cause of packet dropping is that there is no eligible forwarding choice in the neighbourhood table for packet forwarding. But PATH provides the service differentiation and serves different data traffic using dynamic velocity assignment and control trade-off between energy and delay constraint with dynamic power control. Hence, packet dropping is reduced with dynamic performance improving the real-time routing in WSNs.

PATH is the integration of power control mechanism with Two-hop Velocity based Routing (THVR). The key components of this protocol are:

- Forwarding metric and policy, which provides one energy efficient path for forwarding.

- Power adaptation to discover eligible forwarding choices to avoid packet dropping.

- Delay estimator estimates the link delay to update the information for the forwarding policy.

- Initiative drop control module decides whether the packet is to be dropped or not.

It addresses the practical issues like scalability, loss links and network holes. This real time protocol takes into account the energy consumed and the remaining energy of nodes in the path from source to destination thereby balancing energy throughout the network effectively. Performance analysis shows that PATH outperforms THVR in term of dead line miss ratio. Dead line miss ratio (DMR) is decreased in PATH because packets have more time and more suitable path to reach destination. THVR energy metric is based only on the remaining energy of the forwarding candidate but in PATH, it takes into account both remaining energy of the forwarding candidate and energy that are consumed for sending packet to each forwarding node. So, PATH uses energy efficiently than THVR.

\section{Comparison of Real-Time Routing Protocols}

Table 1. Comparison Table of Real-Time Routing Protocols for WSNs

\begin{tabular}{|c|c|c|c|c|c|c|}
\hline & Protocol & $\begin{array}{c}\text { Protocol } \\
\text { Operation }\end{array}$ & Factors Considered & $\begin{array}{l}\text { Performance } \\
\text { metrics }\end{array}$ & $\begin{array}{l}\text { Energy } \\
\text { efficiency }\end{array}$ & Reliability \\
\hline 1 & AODV & $\begin{array}{l}\text { Demand } \\
\text { driven }\end{array}$ & $\begin{array}{l}\text { End-to-End delay, } \\
\text { Packet delivery } \\
\text { fraction and Routing } \\
\text { overhead }\end{array}$ & $\begin{array}{l}\text { End-to- End } \\
\text { delay }\end{array}$ & N/A & N/A \\
\hline 2 & DSR & $\begin{array}{l}\text { Demand } \\
\text { driven }\end{array}$ & $\begin{array}{c}\text { Packet delivery } \\
\text { fraction, end-to-end } \\
\text { delay, Routing } \\
\text { overhead. }\end{array}$ & $\begin{array}{l}\text { Routing } \\
\text { overhead }\end{array}$ & N/A & N/A \\
\hline 3 & RAP & $\begin{array}{l}\text { Query and } \\
\text { Location }\end{array}$ & $\begin{array}{l}\text { Mobility, Dead line } \\
\text { miss ratio and }\end{array}$ & $\begin{array}{c}\text { End-to-End } \\
\text { deadline miss }\end{array}$ & N/A & N/A \\
\hline
\end{tabular}


International Journal of Computer Science \& Engineering Survey (IJCSES) Vol.4, No.3, June 2013

\begin{tabular}{|c|c|c|c|c|c|c|}
\hline & & & Distance constraints & ratio & & \\
\hline 4 & SPEED & Location & $\begin{array}{l}\text { End-to-End delay, } \\
\text { Dead line miss ratio, } \\
\text { Energy } \\
\text { consumption, Void } \\
\text { avoidance }\end{array}$ & $\begin{array}{c}\text { End-to-End } \\
\text { delay, Dead } \\
\text { line miss ratio }\end{array}$ & Low & N/A \\
\hline 5 & $\begin{array}{c}\text { MM } \\
\text { SPEED }\end{array}$ & Location & $\begin{array}{l}\text { Reliability, } \\
\text { Timeliness, } \\
\text { Scalability }\end{array}$ & $\begin{array}{l}\text { Average } \\
\text { delay, } \\
\text { Overhead, } \\
\text { Reliability }\end{array}$ & Low & Good \\
\hline 6 & RPAR & Location & $\begin{array}{c}\text { Energy } \\
\text { consumption, Dead } \\
\text { line miss Ratio, } \\
\text { Lossy links, } \\
\text { scalability, BW } \\
\text { constraints }\end{array}$ & $\begin{array}{c}\text { Energy } \\
\text { Consumption, } \\
\text { Dead line miss } \\
\text { ratio }\end{array}$ & Good & N/A \\
\hline 7 & THVR & Location & $\begin{array}{c}\text { Energy } \\
\text { consumption, } \\
\text { Deadline miss ratio, } \\
\text { Overhead }\end{array}$ & $\begin{array}{c}\text { Energy } \\
\text { consumption, } \\
\text { Deadline miss } \\
\text { ratio }\end{array}$ & High & Good \\
\hline 8 & PATH & Location & $\begin{array}{l}\text { Energy, Power, } \\
\text { Scalability, } \\
\text { Reliability }\end{array}$ & $\begin{array}{c}\text { Energy } \\
\text { consumption, } \\
\text { Deadline miss } \\
\text { ratio }\end{array}$ & High & Good \\
\hline
\end{tabular}

\section{Conclusions}

Many excellent protocols have been developed for WSN's. Due to the time constraints, dynamic node structure, non deterministic nature of the links and resource constrained environment of WSN, real time routing is quite challenging to meet the requirements of RT systems. In this paper the review of real time routing protocols along with their key features, their strengths and weaknesses are presented. The researchers pointed out the various issues like RT, energy aware, end-to-end delay, deadline miss ratio, reliability, scalability and mobility. From the above analysis, it is observed that the issues like energy, delay constraints, QoS and reliability are well addressed. This study reveals that the energy metric is inversely proportional to the time domain in real time application systems.

\section{REFERENCES}

[1] C.Y.Chong, S.P. Kumar," Sensor Networks: evolution, opportunities, and challenges”, Proc. IEEE,vol 91, no.8, pp 923-933, November 2006

[2] T. He. A. Pascal, T. Yan, L. Luo, L. Gu, G. Zhou, R. Stoleru, Q. Cao, J. Stankovic, and T. Abdelzaher, "Achieving real time target tracking using wireless sensor networks", in Proc. IEEE RTAS, pp 37-48, September 2006

[3] D. Malan, T. Fulford Jones, M. Welsh, and S. Moulton, “ An ad hoc sensor network infrastructure for emergency medical care,” in Proc. Int Workshop Wearable implantable body Sensor networks, April 2004.

[4] A. Zhan, T. Xu, G. Ghen, B. Ye and S. Lu, “ A survey on Real-Time Routing Protocols for Wireless Sensor Networks", In Proc. Of the 2nd China Wireless Sensor Network Conference (CWSN-2008), Chongqing, China, October 13-14, 2008. 
International Journal of Computer Science \& Engineering Survey (IJCSES) Vol.4, No.3, June 2013

[5] C.E. Perkins, E.M. Royer, “ Ad hoc On-Demand Distance Vector Routing,” Proc. of 2nd IEEE Workshop on Mobile Computing Systems and Applications, New Orleans, LA, February, 1999, pp 90-100.

[6] C.E. Perkins, E.M. Royer, " Multicast operation of the Ad-hoc On-Demand Distance Vector Routing," Proc. of \%5th annualACM/ IEEE Workshop on Mobile Computing Systems and Applications, pp 207-218, 1999.

[7] David B. Johnson, David A Maltz, Josh Broch "DSR: The dynamic source routing protocol for multihop wireless adhoc networks", in Mobile Computing, Chapter5, pp 153-181, Kluwer Academic publishers, 1996.

[8] C lu, B. M. Blum, T.F.Abdelzaher, J.A. Stankonic and T.He, " RAP : A real time Architecture for large scale wireless sensor networks," in proceedings of RTA S, 2002, September, 2002.

[9] Tian He, John A Stancovic, Chenyang Lu, Tarek Abdelzaher," Speed: A Stateless protocol for real time communication in Sensor network", Proceedings of International Conference on Distributed Computing Systems, Providence, RI, May 2003.

[10] Emad Felemban, Chang Gun lee and Eylem Elcici, "MM SPEED: Multipath Mulit-speed protocol for QoS guarantee of reliability and Timeliness in Wireless sensor networks", IEEE transactions on Mobile computing[J], pp 738-754, 2006.

[11] O. Chipura, Z.He, G.Xing, Q. Chen, Xiaorui Wang, C. Lu, J. Stankovic, T.Abdelzaher, “ Real time Power Aware Routing Protocol in Sensor networks", in the proceedings of IWQoS 2006, June 2006.

[12] Yanjun Li, Chung Shue Chen, Ye-Qiong Song, Zhi wang, Youxian Sun," Enhancing Real-Time Delivery in Wireless Sensor Networks with two-hop information.” , IEEE Transactions on industrial informatics, Vol 5, No. 2, May 2009.

[13] P. Rezayat, M. Mahdavi, M.. Ghasemzadeh, M.Agha.S, “ A Novel Real time Power Aware Routing Protocol”, in the proceedings of IJCSNS, Vol 10, April, 2010.

\section{Authors}

Sandhya Rachamalla is graduated in B.Tech (ECE) from JNTU,Hyd in April, 2004. She completed M.Tech in Digital Systems and Computer Electronics in June, 2009 from JNT University, Ananthapur. Her areas of interest are Wireless Communications, Wireless Sensor Networks and Routing Protocols. She has six publications in various International and National Conferences and Journals. She is presently pursuing Ph.D from JNTU, Hyderabad and working as an Associate Professor in ECE Department of Sreenidhi Institute of Science and Technology, Hyderabad. She is an IEEE member.

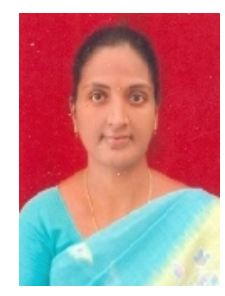

Dr. K. Anitha Sheela is graduated in B. Tech. (ECE) from REC ,Warangal during May,1993 and completed ME in Systems and Signal Processing from College of Engineering, OU, during 1995-1997 and Ph. D from JNTU, Hyderabad during 2003 to 2008. She had worked as Testing Engineer at Onyx Industries for 2 years and has been in teaching for more than a decade. Now, she is the Additional Controller of Examinations at JNTUH, Hyderabad, A.P. India. She has 21 papers published in various National and International Conferences and Journals. Her areas of interest are Wireless Networks, Speech Processing, Pattern Recognition, Image Processing, DSP Processors and Neural Networks. She is Fellow of IETE and life member of ISTE. She

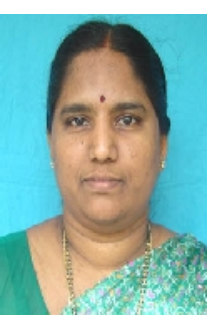
is presently working as an Associate Professor in ECE Dept of JNTU, Hyderabad, guiding 5 Ph.D. scholars .She is Ph. D adjudicator for Sri Satya Sai University, Prasanthi Nilayam. 